\title{
Energy-Efficiency and Spectrum-Efficiency Tradeoff in Coordinated Small-Cell Networks
}

\author{
Xu Zhang, Sheng Zhou, Zhisheng Niu, Xiaokang Lin \\ Tsinghua National Laboratory for Information Science and Technology, \\ Dept. of Electronic Engineering, Tsinghua Univ., Beijing, 100084, P.R. China \\ Email: zhang-xu10@mails.tsinghua.edu.cn, \{sheng.zhou, niuzhs $\} @$ tsinghua.edu.cn, \\ linxk@sz.tsinghua.edu.cn
}

\begin{abstract}
Small-cell networks play an important role in next generation mobile networks. Multiple antennas can be employed at the small-cell base stations (SBS) to further improve the spectrum-efficiency. However, the increased number of antenna elements induces more energy consumption, i.e., there exists a tradeoff between the energy-efficiency and the spectrumefficiency. In this paper, the network performance is analyzed which takes the coordinated scheduling into account. The coordination relationship among neighboring SBSs is modeled by a coordination graph. The coordinated scheduling is achieved by finding the maximum independent set of the graph. By deriving the average size of the maximum independent set, the network throughput is obtained. Simulation results validate the derivation. Then, the tradeoff between the energy-efficiency and the spectrum-efficiency is analyzed. It is found that the singleantenna SBSs are not energy-efficiency as the density of SBSs increases.
\end{abstract}

Index Terms-Energy efficiency, spectrum efficiency, small cell, inter-cell coordination, random graph

\section{INTRODUCTION}

The next evolution of mobile networks has a paradigm shift in the design criteria, such as higher spectrum-efficiency (SE), higher energy-efficiency (EE), lower latency, and supporting increased number of mobile devices (including mobile users and machines). Traditionally, the deployment of homogeneous macro base stations (BS) is the efficient way to improve the SE. However, it is difficult for operators to further increase the density of macro BSs due to the ever-increasing cost of the site acquisition and the huge amount of the energy consumption. To solve this problem, the low-cost small cells, such as femtocells, relays, and distributed antennas are densely deployed within the coverage of a macrocell, to improve the $\mathrm{SE}$ by reducing the distance from the BSs to the users. The resultant network is commonly referred to a heterogenous networks (HetNets) which play an important role in the next generation mobile networks [1] [2].

Besides, multiple antennas can be employed at the small-cell base stations (SBS) to improve the spatial reuse by generating a narrow beam such as the massive MIMO techniques. The SE will be further increased. However, the increased number of antennas consumes more circuit power. There is a tradeoff between the EE and the SE. Previously, the EE is analyzed in the cases with a single BS or regularly located BSs [3]- [5]. However, it is difficult to extend the previous works to the case of small cells. In the HetNets, the deployment of small cells is commonly demand-driven and achieved by the endusers which leads to a dense and irregular network topology. Moreover, the performance analysis based on the regulartopology model such as the grid-based model is intractable as the network size growth [6].

Recently, a tractable model is proposed by Andrews et al. in which BSs are modeled as a spatial Poisson Point Process (PPP) [7]. The EE of the small-cell networks with multipleantenna SBSs is analyzed based on this model [8]. However, the analysis model treats the inter-cell interference as noise, i.e., the interference management is not considered. To our best knowledge, due to the analytical difficulty of the interference distribution with the cooperative BSs, the EE performance of the small cells with inter-cell coordination is still unknown.

In this paper, we analyze the downlink performance with coordinated scheduling among different small cells. The coordination is modeled from users' point of view who determine whether the neighboring SBSs should join the coordination. Specifically, each SBS has a coordination region which is related to the location of the corresponding receiver. If the neighboring SBS locates within this region, this neighboring SBS should coordinate. With the coordination relationship, a random coordination graph can be generated. The coordinated scheduling can be seen as finding the maximum independent set of the coordination graph. By deriving the average size of the maximum independent set, the network performance is obtained. The derivation is verified by the simulation results. Consequently, the tradeoff between the EE and the SE is obtained.

The rest of this paper is organized as follows. System model is described in section II. In section III, the intercell coordination is introduced from the users' point of view and the area spectrum-efficiency and area energy-efficiency is defined. Section IV shows the analysis framework which derives the average size of the maximum independent set in the coordination graph. Simulation results are shown in section V. Finally, section VI concludes the paper.

\section{System Model}

\section{A. Network Topology}

There are $N$ SBSs which are uniformly located in a circular area with the radius of $R$. Each SBS has $M$ antennas and provides a coverage of radius $D$. Single-antenna users are 


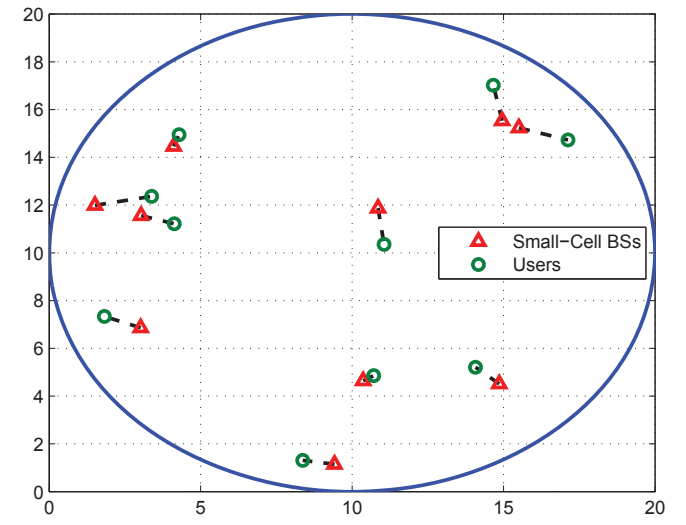

Fig. 1. The network topology with small cells and their corresponding receivers. The radius of the area is $R=10$ unit area. The coverage radius of each small-cell BS is $D=2$ unit area. There are total 10 small-cell BSs.

uniformly distributed around each SBS. For each transmission, there are total $N$ links denoted by $\left\{l_{1}, l_{2}, \ldots, l_{N}\right\}$. Since users are uniformly distributed around each SBS, the distance between the SBS and the corresponding receiver is a random variable. Given the transmission direction, the distribution is given by $f_{D}(r)$,

$$
f_{D}(r)=\frac{2 r}{D^{2}}, r \in[0, D] .
$$

The transmission direction of each SBS is uniformly distributed within $[0,2 \pi]$.

Given link $l_{n} \in\left\{l_{1}, l_{2}, \ldots, l_{N}\right\}$, the transmitter of $l_{n}$ is denoted by $\mathrm{B}_{n}$ and the receiver is denoted by $\mathrm{UE}_{n}$. With polar coordinates, the location of $\mathrm{B}_{n}$ is denoted by $\left(r_{\mathrm{T}, n}, \theta_{\mathrm{T}, n}\right)$ where $n \in\{1,2, \ldots, N\}$. Since the distribution of the SBSs' location is independent and identical, the distribution of $\left(r_{\mathrm{T}, n}, \theta_{\mathrm{T}, n}\right), \forall n \in\{1, \ldots, N\}$, is given by

$$
f_{\mathrm{T}}(r, \theta)=\frac{r}{\pi R^{2}}, r \in[0, R], \theta \in[0,2 \pi] .
$$

Accordingly, the location of $\mathrm{UE}_{n}$ is denoted by $\left(r_{\mathrm{R}, n}, \theta_{\mathrm{R}, n}\right)$ where $n \in\{1,2, \ldots, N\}$. Given the link distance as $d$, the distribution of $\left(r_{\mathrm{R}, n}, \theta_{\mathrm{R}, n}\right), \forall n \in\{1,2, \ldots, N\}$, is given by

$$
f_{\mathrm{R}}(r, \theta)=\frac{r}{\pi R^{2}} \frac{f_{\phi}(r, d, R)}{2 \pi}, r \in[0, d+R], \theta \in[0,2 \pi],
$$

where $f_{\phi}\left(r, d, r_{0}\right)$ is a function of $r$ which is given by

$$
f_{\phi}\left(r, d, r_{0}\right)=\left\{\begin{array}{l}
2 \pi, \quad 0<r<\max \left(r_{0}-d, 0\right) \\
2 \arccos \left(\frac{r^{2}+d^{2}-r_{0}^{2}}{2 d r}\right), \\
\quad \max \left(r_{0}-d, d-r_{0}\right) \leq r \leq r_{0}+d \\
0, \text { else }
\end{array} .\right.
$$

\section{B. Antenna Pattern}

In this section, the radiation pattern is modeled by a flattop antenna beam which is commonly used to characterize the radiation pattern of the antenna array [9]. The antenna gain $g(\phi)$ is given by

$$
g(\phi)=\left\{\begin{array}{l}
G_{\mathrm{ml}},|\phi| \leq \frac{\theta_{\mathrm{BW}}}{2} \\
G_{\mathrm{sl}}, \text { else }
\end{array},\right.
$$

where $\phi$ represents the azimuthal angle, $\theta_{B W}, G_{\mathrm{ml}}$ and $G_{\mathrm{sl}}$ represent the beamwidth, the transmission gain of the main lobe and the transmission gain of the side lobe, respectively. Since all links are assumed on the same horizontal plane, the variation of the beam pattern over the elevation angle is not considered.

In order to obtain those parameters, an uniformly distributed linear-array is assumed. The interval distance between each elements is $\lambda / 2$, where $\lambda$ represents the wave length. The radiation pattern of an uniformly distributed linear-array consists of two parts, the pattern of the antenna element itself, and the pattern of the array with the actual elements replaced by isotropic point sources, which are called the element pattern and the array factor respectively. The total pattern of the array is the product of the element pattern and the array factor.

With the far field approximation, the array factor is a function of the azimuthal angle which is

$$
\mathrm{AF}(\phi)=\mathbf{w}^{\mathrm{H}} \mathcal{G}(\phi)=\sum_{n=1}^{M} w_{n} e^{j(n-1)\left(k \frac{\lambda}{2} \cos \phi\right)},
$$

where $k=\frac{2 \pi}{\lambda}$ is the wavenumber, $\left\{w_{n} \in \mathbb{C}, n=1, \ldots, M\right\}$ are complex values for the beamforming and $\mathcal{G}(\phi)=$ $\left\{1, e^{j\left(k \frac{\lambda}{2} \cos \phi\right)}, e^{j 2\left(k \frac{\lambda}{2} \cos \phi\right)}, \ldots e^{j(M-1)\left(k \frac{\lambda}{2} \cos \phi\right)}\right\}$ denotes the array steering factor. The antenna elements are assumed as the isotropic point sources, then element pattern is given by

$$
\mathbf{E}_{e}(\phi)=1, \forall \phi \in[0,2 \pi] .
$$

Accordingly, the free-space radiation pattern is given by

$$
F(\phi)=\operatorname{AF}(\phi) \mathbf{E}_{e}(\phi) .
$$

The directivity of the array at free space is denoted by $\mathcal{D}$ and is given by

$$
\mathcal{D}(\phi)=\frac{F(\phi) F(\phi)^{\mathrm{H}}}{\frac{1}{2 \pi} \oint F(\phi) F(\phi)^{\mathrm{H}} d \phi} .
$$

where $(\cdot)^{\mathrm{H}}$ denotes the operator of complex conjugate transpose (Hermitian).

Therefore, the beamforming gain is given by

$$
G(\phi)=\eta_{\mathrm{T}} \mathcal{D}(\phi),
$$

where $\eta_{\mathrm{T}}=1$ represents the array efficiency.

Accordingly, the value of $G_{\mathrm{ml}}$ is given by

$$
G_{\mathrm{ml}}=\max _{\phi \in[0,2 \pi]} G(\phi) .
$$

$G_{\mathrm{sl}}$ is obtained by calculating the power gain of the first side lobe. The beamwidth $\theta_{\mathrm{BW}}$ is defined as the half-power beamwidth (HPBW) which is

$$
\theta_{\mathrm{BW}}=\left|\phi_{\mathrm{HPBW}, \text { left }}-\phi_{\mathrm{HPBW}, \text { right }}\right|,
$$

where $G\left(\phi_{\mathrm{HPBW}, \text { left }}\right)=G\left(\phi_{\mathrm{HPBW}, \text { right }}\right)=\frac{1}{2} \max (G(\phi))$. 


\section{Beamforming}

Consider the case that the SBSs are commonly deployed on top of the user clusters. When an uniformly distributed lineararray is used at the SBS, the received signal at the $\mathrm{UE}_{n}$ is given by [10]

$$
y_{n}=\mathbf{h}_{n}^{\mathrm{T}} \mathbf{R}_{\mathrm{T}}^{\frac{1}{2}} \mathbf{w} x_{n}+n_{0},
$$

where $(\cdot)^{\mathrm{T}}$ denotes the operator of matrix transpose, $\mathbf{h} \in$ $\mathbb{C}^{M \times 1}$ is an independent and identically distributed channel vector such that $\mathbf{h} \sim \mathcal{C N}\left(0, \mathbf{I}_{M}\right)$, w $\in \mathbb{C}^{1 \times M}$ represents the beamforming vector, $\mathbf{w}^{\mathrm{H}} \mathbf{w}=1 . n_{0} \sim \mathcal{C N}(0,1)$ is the thermal noise at the receiver side. $\mathbf{R}_{\mathrm{T}} \in \mathbb{C}^{M \times M}$ is the transmit correlation matrix and given by

$$
\left(\mathbf{R}_{\mathrm{T}}\right)_{i j}=\frac{\left(\mathbf{R}_{\mathrm{C}}\right)_{i j}}{\sqrt{\left(\mathbf{R}_{\mathrm{C}}\right)_{i i}\left(\mathbf{R}_{\mathrm{C}}\right)_{j j}}}=\frac{\left(\mathbf{R}_{\mathrm{C}}\right)_{i j}}{\oint A(\phi) d \phi},
$$

where $\mathbf{R}_{\mathrm{C}}=\oint \mathcal{G}(\phi) \mathcal{G}^{\mathrm{H}}(\phi) A(\phi) d \phi$, and $A(\phi)$ represents the channel angular power spectrum. For a Gaussian cluster of the scatterers with HPBW of $\sigma_{A} \sqrt{2 \ln 2}, A(\phi)$ is given by

$$
A(\phi)=\frac{1}{\sqrt{2 \pi} \sigma_{A}} \exp \left(-\frac{\phi^{2}}{2 \sigma_{A}^{2}}\right) .
$$

The beamforming vector is obtained by maximizing the receiving SNR (Signal-Noise-Ratio), then the beamforming vector is the eigenvector corresponding to the maximum eigenvalue of the $\mathbf{R}_{\mathrm{T}}$,

$$
\mathbf{w}^{\text {opt }}=\arg \max _{\mathbf{w} \in \mathbb{C}^{M \times 1}} \mathbf{w}^{\mathrm{H}} \mathbf{R}_{\mathrm{T}} \mathbf{w} .
$$

\section{Power Consumption Model}

In this paper, the total power consumption is given by [11]

$$
P_{\text {total }, \mathrm{BS}}(m)=\frac{P_{\mathrm{T}}}{\xi}+m P_{\text {Ant }}+P_{0},
$$

where $m$ represents the number of antenna elements at each base station, $P_{0}$ denotes the power consumption which is independent with the transmissions, $P_{\text {Ant }}$ denotes the total power consumption which comes from the circuits, $P_{\mathrm{T}}$ denotes the output power of the transmitter and $\xi$ is the efficiency of the power amplifier. In order to focus on the effects of the number of antenna elements, $P_{\mathrm{T}}$ is a constant value.

Suppose there are $N^{\prime}$ SBSs transmit simultaneously and each with $m$ antennas, the power consumption of the whole network is given by

$$
P_{\mathrm{Net}}=N^{\prime} P_{\text {total }, \mathrm{BS}}(m)+N P_{0} .
$$

\section{INTER-CELL COORDINATION}

\section{A. Coordination Region}

In order to characterize the behavior of inter-cell coordination, we define the Coordination Region (CR) as shown in Fig. 2 with the center at the location of the receiver. The total region is divided into two parts: Inner-Region and Outer-Region.

1. Inner-Region: Every SBS who is located within this region should coordinate with $\mathrm{B}_{n}$.

2. Outer-Region: Only if the $\mathrm{UE}_{n}$ locates within the transmission direction of the SBS who is located within this outer region, the SBS coordinates with $\mathrm{B}_{n}$. Otherwise, it is not.

The radius of these two regions are denoted as $r_{\mathrm{CR}, 1}$ and $r_{\mathrm{CR}, 2}$ respectively, and $r_{\mathrm{CR}, 1}>r_{\mathrm{CR}, 2}$. Since the signal attenuation is modeled by the free-space model and the thermal noise is ignorable in the case of high-dense small cells, then

$$
\begin{aligned}
& r_{\mathrm{CR}, 1}=\left(\frac{\eta P_{\mathrm{T}} G_{\mathrm{T}} G_{\mathrm{R}}}{I_{\mathrm{req}}}\right)^{\frac{1}{\alpha}}, \\
& r_{\mathrm{CR}, 2}=\left(\frac{\eta P_{\mathrm{T}} G_{\mathrm{sl}} G_{\mathrm{R}}}{I_{\mathrm{req}}}\right)^{\frac{1}{\alpha}},
\end{aligned}
$$

where $\eta$ is the path-loss coefficient, $\alpha$ is the attenuation coefficient, $G_{\mathrm{T}}=G_{\mathrm{ml}}$ and $G_{\mathrm{R}}=1$ are the antenna gain of transmitter and receiver respectively, $I_{\text {req }}$ is the maximum tolerable interference at the receiver which is given by

$$
I_{\text {req }}=\eta \frac{P_{\mathrm{T}} G_{\mathrm{T}} G_{\mathrm{R}} d^{-\alpha}}{\gamma_{0}},
$$

where $d$ is the transmission distance of the given link $l_{n}$ and $\gamma_{0}$ is the required SNR. Then, plugging (21) into (19) and (20), we have $r_{\mathrm{CR}, 1}(d)=\gamma_{0}^{\frac{1}{\alpha}} d$ and $r_{\mathrm{CR}, 2}(d)=\left(\frac{G_{\mathrm{sl}} \gamma_{0}}{G_{\mathrm{ml}}}\right)^{\frac{1}{\alpha}} d$. It is can be seen that the coordination region related to the link distance. Since the transmitting power is a constant, the farlocated user should have larger coordination region to ensure its link quality.

\section{B. Problem Formulation}

For coordination scheduling, only those SBSs who do not belong to the same coordination group can be scheduled simultaneously. If the maximum number of concurrent links are scheduled at each time, the system achieves the maximum throughput.

Therefore, the Spectrum-Efficiency (SE) is defined as

$$
\mathrm{SE}=\frac{N^{\prime} c}{A W},
$$

where $N^{\prime}$ represents the average number of the maximum concurrent links with respect to the random topologies and $A$ represents the area of the whole network, $c=W \log _{2}\left(1+\gamma_{0}\right)$ is the data rate of a given link, and $W$ is the bandwidth.

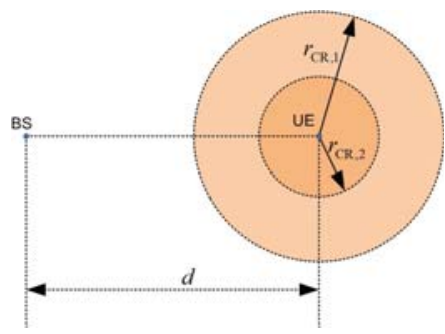

Fig. 2. The Coordination Region of SBS, the outer region with radius of $r_{\mathrm{CR}, 1}$ is for the neighboring SBSs who point to the UE, the inner region with radius of $r_{\mathrm{CR}, 2}$ is for every neighboring SBSs. Once the other SBSs locate in those regions, they should coordinate their transmissions with this link. 


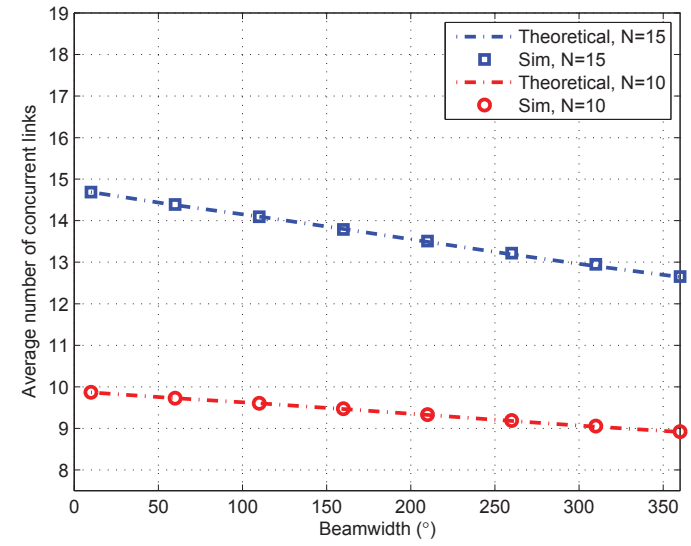

Fig. 3. The number of concurrent links with different number of SBSs, where $N$ represents the total number of SBSs. The region is circular with radius of 20 unit length, and the coverage area of each SBS is $D=2$ unit length. The Coordination Region of each link is set as $r_{\mathrm{CR}, 1}(d)=2 d, r_{\mathrm{CR}, 2}(d)=0.5 d$.

Accordingly, the Energy-Efficiency (EE) is defined as the ratio between the total data rate and the total energy consumption over the whole network which is given by

$$
\mathrm{EE}=\frac{1}{A} \frac{N^{\prime} c}{N^{\prime} P_{\text {total, BS }}+N P_{0}} .
$$

\section{Performance Analysis}

In order to analyze the system performance, the graph theory is used. For each random topology, there are total $N$ links, the links are defined as vertexes of the coordination graph. The coordination relationship is interpreted by the edges of the graph. When the $\mathrm{B}_{1}$ locates within the $\mathrm{CR}$ of link $l_{2}$, then there exists an edge between those two vertexes, $l_{1}$ and $l_{2}$. The independent set is the set of vertexes in which there exists no edges connecting any of two vertexes. The maximum independent set is the largest independent set.

In order to derive the average size of the maximum independent set, define the probability $P_{n}(v)$ as the probability that the size of maximum independent set equals to $v$ when the total number of links is $n$, where $v \in\{1,2, \ldots, n\}$. Then, $P_{1}(1)=1$. Denote the probability of two links do not locate at the CR of each other by $Q$. Then, we have $P_{2}(1)=1-Q$ and $P_{2}(2)=Q$. Let $\mathbf{P}_{n}^{\mathrm{T}}=\left\{P_{n}(1), P_{n}(2), \ldots, P_{n}(n)\right\}$,

$$
\mathbf{P}_{n}=\mathbf{H}_{n} \mathbf{H}_{n-1} \cdots \mathbf{H}_{2} \mathbf{H}_{1}=\prod_{t=1}^{n} \mathbf{H}_{t}
$$

where $\mathbf{H}_{n} \in \mathcal{R}^{n \times(n-1)}$, and

$$
\mathbf{H}_{n}(i, j)=\left\{\begin{array}{l}
(1-Q)^{n-1}, i=1, j=1 \\
1-(1-Q)^{n-1}, i=2, j=1 \\
1-Q^{j}, i=j, j \neq 1 \\
Q^{j}, i=j+1, j \neq 1 \\
0, \text { else }
\end{array} .\right.
$$

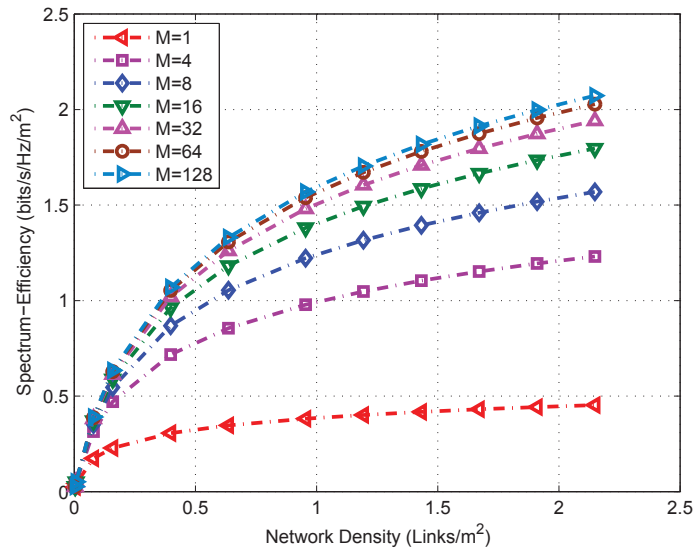

Fig. 4. The Spectrum-Efficiency versus the network Density.

The calculation of $\mathbf{P}_{n}$ is based on the idea of checking all of the links by whether the maximum independent set with newly added link is still an independent set. The maximum independent set is selected iteratively until all of the links have been checked [12]. Note that the calculation is an approximation. When the coverage area by SBSs is relatively small, it achieves an accurate performance which is shown by simulation results.

For the calculation of $Q$, consider the case with two links denoted by $\left\{l_{1}, l_{2}\right\}$. Since $Q$ denotes the probability of two links do not locate at the $\mathrm{CR}$ of each other, $Q$ is obtained by the inclusion-exclusion rule of the probability,

$$
\begin{aligned}
Q= & 1-\operatorname{Pr}\left\{l_{1} \rightarrow l_{2}\right\}-\operatorname{Pr}\left\{l_{2} \rightarrow l_{1}\right\} \\
& +\operatorname{Pr}\left\{l_{1} \rightarrow l_{2}, l_{2} \rightarrow l_{1}\right\},
\end{aligned}
$$

where $\operatorname{Pr}\left\{l_{1} \rightarrow l_{2}\right\}$ represents the probability that the transmitter $\mathrm{B}_{1}$ locates within the $\mathrm{CR}$ of link $l_{2}, \operatorname{Pr}\left\{l_{2} \rightarrow l_{1}\right\}$ is the probability that the transmitter $\mathrm{B}_{2}$ locates within the $\mathrm{CR}$ of link $l_{1}$, and $\operatorname{Pr}\left\{l_{1} \rightarrow l_{2}, l_{2} \rightarrow l_{1}\right\}$ represents the probability that the transmitter $\mathrm{B}_{1}$ locates within the $\mathrm{CR}$ of link $l_{2}$ and, at the same time, the transmitter $B_{2}$ locates within the $C R$ of link $l_{1}$. Due to the limited length of the paper, the detail of the calculation is omitted.

Then, the average size of the maximum independent set is derived accordingly, $N^{\prime}=\sum_{n=1}^{N} n P_{N}(n)$.

\section{Simulation Results}

The simulation parameters are set as follows: the radius of the area is $R=20$ unit length. Users are uniformly distributed around each SBS within the radius of $D=2$ unit length. The path-loss factor is given by $\alpha=4$. The bandwidth is $B W=10 \mathrm{MHz}$. The power consumption is $P_{\mathrm{T}}=6.3 \mathrm{~W}$, $P_{\text {Ant }}=35 \mathrm{~W}, P_{0}=34 \mathrm{~W}$ and $\xi=0.32$ [8]. The angle spread is set to $\sigma_{A}=20^{\circ}$. The minimum required SNR is $\gamma_{0}=100$. In order to validate the calculation results, we assume that $r_{\mathrm{CR}, 1}(d)=2 d$ and $r_{\mathrm{CR}, 2}(d)=0.5 d$ which are the functions of the transmission distance $d$. The average number of concurrent 
links versus the beamwidth is plotted in Fig. 3. It shows that the theoretical results match with the simulation results.

In Fig. 4, the SE versus the network density is plotted. The SE monotonically increases with the network density given the number of antennas. It is because that, with the coordinated scheduling, the average number of maximum concurrent links always increases as the density increases. Given the density, the SE increases as the number of antennas increases. It is because that the spatial reuse increases with the smaller beamwidth of each link.

The EE versus the density of SBSs is plotted in Fig. 5. Given the number of antennas, the EE monotonically decreases over the density of SBSs. It is because that the increased spatial reuse always smaller than the increased density. It indicates that energy-efficiency can not be improved by only increasing the density since it induces more transmitting-independent energy consumption. Given the density, the EE decreases with the number of antennas when the density is relatively low. In that case, single-antenna SBS achieves the maximum EE. However, as the density increases, EE is a concave function of the number of antennas. There exists an optimal number of antennas which is larger than one.

From Fig. 4 and 5, the tradeoff between EE and SE can be seen. In the low density case, the EE always decreases with the SE. However, in the high density case, the EE firstly increases, then it decreases, when the SE increases.

\section{CONCLUSIONS}

The energy-efficiency of randomly deployed small-cell with coordinated scheduling is analyzed. Simulation results validates the theoretical analysis. Based on the theoretical results, the tradeoff between energy-efficiency (EE) and spectrumefficiency (SE) is analyzed. The EE is a decreasing function of the SE when the density of the SBSs is relatively low. In the high-density case, the EE firstly increases, then it decreases, when the SE increases. Moreover, given the number of antennas, the energy-efficiency is a decreasing function of the density. When the density of SBSs is relatively low,

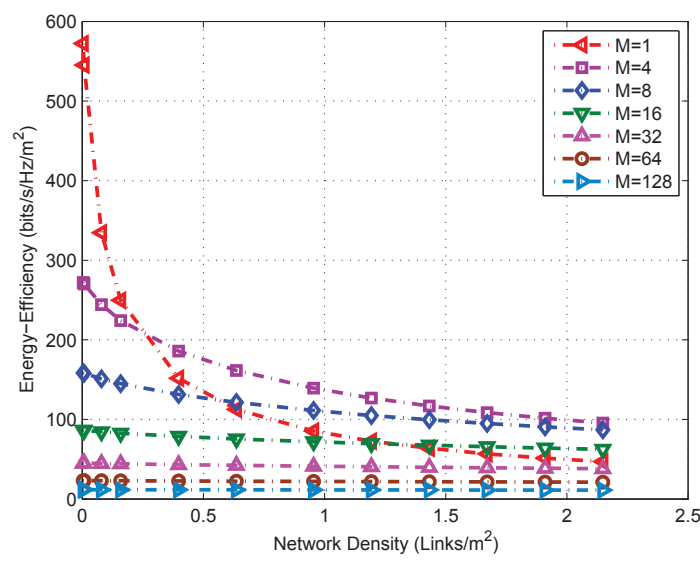

Fig. 5. The Energy-Efficiency versus the network density. single-antenna base stations achieves the maximum energyefficiency. As the density of SBSs increases, the maximum energy-efficiency is achieved by the SBS with more than one antennas.

\section{ACKNOWLEDGMENT}

This work is sponsored in part by the National Basic Research Program of China (973 Program: 2012CB316001), the National Science Foundation of China (NSFC) under grant No. 61201191, the Creative Research Groups of NSFC under grant No. 61321061, Hitachi R\&D Headquarter.

\section{REFERENCES}

[1] J. Xu, J. Wang, Y. Zhu, Y. Yang, X. Zheng, S. Wang, L. Liu, K. Horneman, and Y. Teng, "Cooperative distributed optimization for the hyper-dense small cell deployment," IEEE Communications Magazine, vol. 52, no. 5, pp. 61-67, May 2014.

[2] Y.A. Sambo, M.Z. Shakir, K.A. Qaraqe, E. Serpedin, and M.A. Imran, "Expanding cellular coverage via cell-edge deployment in heterogeneous networks: spectral efficiency and backhaul power consumption perspectives," IEEE Communications Magazine, vol. 52 , no. 6, pp. 140-149, Jun. 2014.

[3] Z. Xu, C. Yang, G. Y. Li, S. Zhang, Y. Chen, and S. Xu, "Energyefficient configuration of spatial and frequency resources in MIMOOFDM systems," IEEE Trans. Commun., vol. 61, no. 2, Feb. 2013.

[4] E. Bjornson, L. Sanguinetti, J. Hoydis, and M. Debbah, "Designing multiuser MIMO for energy efficiency: when is massive MIMO the answer?," in Proc. IEEE Wireless Communications and Networking Conference (WCNC 2014), Istanbul, Turkey, Apirl 2014.

[5] O. Onireti, F. Heliot, M.A. Imran, "On the energy efficiency-spectral efficiency trade-off of distributed MIMO systems," IEEE Trans. Communications, vol. 61, no. 9, pp. 3741-3753, Sept. 2013.

[6] J. Xu, J. Zhang and J. Andrews, "On the accuracy of the Wyner model in cellular networks," IEEE Trans. Wireless Communications, vol. 10, no. 9, pp. 3098-3134, Nov. 2011.

[7] J.G. Andrew, F. Baccelli, and R.K. Ganti, "A tractable approach to coverage and rate in cellular networks," IEEE Trans. Communications, vol. 59, no. 11, pp.3122-3134, Nov. 2011.

[8] C. Li, J. Zhang, and K.B. Letaief, "Throughput and energy efficiency analysis of small cell networks with multi-antenna base stations," IEEE Trans. Wireless Communications, vol. 13, no. 5, pp. 2505-2517, May 2014.

[9] S. Singh, R. Mudumbai, and U. Madhow, "Interference analysis for highly directional $60-\mathrm{GHz}$ mesh networks: the case of rethinking medium access control," IEEE/ACM Trans. Networking, vol. 19, no. 5, pp. 1513-1527, Oct. 2011

[10] O.N. Alrabadi, E. Tsakalaki, H. Huang and G.F. Pedersen, "Beamforming via large and dense antenna arrays above a clutter," IEEE Journal on Selected Area in Communications, vol. 31, no. 2, pp. 314-325, Feb. 2013

[11] S. Cui, A.J. Goldsmith, A. Bahai, "Energy-efficiency of MIMO and cooperative MIMO techniques in sensor networks," IEEE Journal on Selected Areas in Communications, vol.22, no.6, pp. 1089-1098, Aug. 2004.

[12] L.X. Cai, L. Cai, X. Shen and J.W. Mark, "Rex: a randomized exclusive region based scheduling scheme for mmWave WPANs with directional antenna," IEEE Trans. Wireless Communications, vol. 9, no. 1, pp. 113 121, Jan. 2010. 